\title{
Discovery and identification of infrared counterpart candidates of four Galactic centre low mass X-ray binaries ${ }^{\star}$
}

\author{
P. A. Curran ${ }^{1}$, S. Chaty ${ }^{1}$, and J. A. Zurita Heras ${ }^{2}$ \\ ${ }^{1}$ Laboratoire AIM, CEA/IRFU-Université Paris Diderot-CNRS/INSU, CEA DSM/IRFU/SAp, Centre de Saclay, \\ 91191 Gif-sur-Yvette, France \\ 2 François Arago Centre, APC, Université Paris Diderot, CNRS/IN2P3, CEA/DSM, Observatoire de Paris, 13 rue Watt, \\ 75205 Paris Cedex 13, France
}

Received 6 May 2011 / Accepted 1 July 2011

\begin{abstract}
Context. The near infrared (nIR)/optical counterparts of low mass X-ray binaries (LMXBs) are often observationally dim and reside in high source density fields which make their identification problematic; however, without such a counterpart identification we are unable to investigate many of the properties of LMXB systems.

Aims. Here, in the context of a larger identification campaign, we examine the fields of four LMXB systems near the Galactic centre, in a bid to identify nIR/optical counterparts to the previously detected X-ray point sources.

Methods. We obtain nIR/optical images of the fields with the ESO - New Technology Telescope and apply standard photometric and astrometric calibrations; these data are supplemented by Spitzer-GLIMPSE catalog data.

Results. On the basis of positional coincidence with the arcsecond accurate X-ray positions, we identify unambiguous counterpart candidates for XTE J1637-498, IGR J17379-3747, IGR J17585-3057 and GX 9+1.

Conclusions. We propose tentative nIR counterparts of four LMXBs which require further investigation to confirm their associations to the X-ray sources.
\end{abstract}

Key words. X-rays: binaries - infrared: stars - X-rays: individuals: XTE J1637-498 - X-rays: individuals: IGR J17379-3747 X-rays: individuals: IGR J17585-3057 - X-rays: individuals: GX 9+1

\section{Introduction}

Given the main sequence or red giant nature of the companion stars of low mass X-ray binaries (LMXBs), they are intrinsically dim in the optical and near-infrared (nIR), and hence difficult to detect (see e.g., Charles \& Coe 2006 for a review of the optical and nIR properties of LMXBs). The intrinsic dimness of all these sources is further compounded by the high level of optical extinction in the direction of the Galactic plane (Schlegel et al. 1998), where many LMXBs are found (Grimm et al. 2002); hence observing at nIR wavelengths, where this is less pronounced, may increase the chance of a detection. Due to their positions in the Galactic plane or centre, where the optical/nIR (OIR) source density is extremely high, accurate X-ray or radio positions are necessary to confidently identify the OIR counterparts of LMXBs and reduce the probability of chance superpositions. The required (sub)arcsecond positions are now more readily available due to missions such as Swift, XMM-Newton and Chandra, and when combined with the current generation of optical telescopes and instruments, increase the likelihood of counterpart discovery.

If the LMXB is a persistent source or a transient source in outburst (van Paradijs \& McClintock 1994) emission from the active accretion disk may be brighter than the companion star

* Based on observations collected at the European Organisation for Astronomical Research in the Southern Hemisphere, Chile under ESO programs 081.D-0401 \& 084.D-0535 (P.I. Chaty). and more likely to be detected at OIR wavelengths; at nIR wavelengths it is even possible that a jet component will make a significant contribution to the emission (e.g., Russell \& Fender 2010). Broadband spectral energy distribution (SED) fitting of observed OIR counterparts can allow the various emission components (companion star and accretion disk/jet if present) to be disentangled from each other and, along with spectral lines, allows the spectral type - and hence mass - of the companion to be constrained. When combined with radial velocity information from X-ray or optical light curves this allows constraints to be placed on the mass of the compact object with which to confirm the nature of the compact object as derived from X-ray spectral or timing properties. Furthermore the relationship between the OIR and X-ray flux can be used as a diagnostic with which to derive the compact object or companion star nature, and to identify the state of the system at the time of observations (e.g., Russell et al. 2006).

Here we examine the fields of four LMXB systems near the Galactic centre, in a bid to identify nIR/optical counterparts to the previously detected X-ray point sources (Table 1). For XTE J1637-498， IGR J17379-3747， IGR J17585-3057 and GX9+1 we identify unambiguous counterpart candidates on the basis of positional coincidence with the arcsecond accurate X-ray positions. We have also observed a further three LMXB systems (1A 1742-294, SLX 1744-300 and IGR J17505-2644) ${ }^{\star}$ as part of our campaign to identify the nIR counterparts of high energy sources near the Galactic centre. Though we do not detail these observations, they confirm the 
Table 1. X-ray and nIR positions for the LMXBs in our study.

\begin{tabular}{lcccccc}
\hline \hline & X-ray & & & nIR & & \\
Source & RA & Declination & Err $\left({ }^{\prime \prime}\right)$ & RA & Declination & Err $\left({ }^{\prime \prime}\right)$ \\
\hline XTE J1637-498 & $16: 37: 02.67$ & $-49: 51: 40.6$ & 1.8 & $16: 37: 02.66$ & $-49: 51: 40.0$ & 0.16 \\
IGR J17379-3747 & $17: 37: 58.81$ & $-37: 46: 19.6$ & 1.4 & $17: 37: 58.77$ & $-37: 46: 19.9$ & 0.32 \\
IGR J17585-3057 & $17: 58: 29.85$ & $-30: 57: 01.6$ & 0.6 & $17: 58: 29.83$ & $-30: 57: 01.6$ & 0.16 \\
GX 9+1 & $18: 01: 32.15$ & $-20: 31: 46.1$ & 0.6 & $18: 01: 32.15$ & $-20: 31: 46.2$ & 0.16 \\
\hline
\end{tabular}

References. To X-ray positions: ${ }^{1}$ Starling et al. (2008), ${ }^{2}$ Krimm et al. (2008), ${ }^{3}$ M. Revnivtsev (2011, priv. comm.).

Table 2. Observation log.

\begin{tabular}{lcc}
\hline \hline Source & Night of observation & Filters \\
\hline XTE J1637-498 & September 9, 2008 & optical/nIR \\
& September 20, 21 2008 & optical, nIR \\
IGR J17379-3747 & September 29, 2008 & optical/nIR \\
& October 2, 3 2008 & optical, nIR \\
IGR J17585-3057 & March 29, 2010 & $K_{\mathrm{S}}$ \\
GX 9+1 & March 28, 2010 & $K_{\mathrm{S}}$ \\
\hline
\end{tabular}

Notes. The second epoch of observations, if done, were carried out over 2 nights: optical on the first, nIR on the second.

results of Zolotukhin \& Revnivtsev (2011) and have been used to verify our reduction methods. In Sect. 2 we introduce our observations and reduction method while in Sect. 3, after briefly introducing each source, we detail the results of those observations. We summarise our findings in Sect. 4. Throughout, positions (J2000) are given with $90 \%$ confidence while all others values, including magnitudes, are given with $1 \sigma$ confidence.

\section{Observations and data analysis}

Optical $(U, B, V, R, i)$ and $\mathrm{nIR}\left(J, H, K_{\mathrm{S}}\right)$ data were obtained with the ESO Faint Object Spectrograph and Camera (v.2; EFOSC2) and the Son of ISAAC (SofI) infrared spectrograph and imaging camera on the $3.58 \mathrm{~m}$ ESO - New Technology Telescope (NTT) over two observing runs in September/October 2008 and March 2010 (Table 2). The NTT data used a dithered pattern of 3 and 9 exposures per final image in the optical and nIR respectively. Data were reduced using the IRAF package wherein crosstalk correction, flatfielding, sky subtraction, biassubtraction and frame addition were carried out as necessary. The $3.9^{\prime} \times 3.9^{\prime}$ images were astrometrically calibrated against 2MASS (Skrutskie et al. 2006) or USNO-B1.0 (Monet et al. 2003) within the GAIA package and given positional errors include a $0.16^{\prime \prime}$ MASS systematic uncertainty (Table 1).

PSF photometry was carried out on the final images using the DAOPHOT package (Stetson 1987) within IRAF. The magnitude of the source of interest in each field was calculated relative to a number of comparison stars in the field, including the scatter as a measure of error. The comparison stars were calibrated against Persson et al. (1998) or Landolt (1992) photometric standards (though in the case of IGR J17379-3747, no suitable optical standards were observed so magnitudes were calibrated against USNO-B1.0 sources). If a field was observed on more than one night we tested for variability, using only the error associated with the relative magnitude. As no variability was found, the tabulated magnitudes are calculated from the weighted average relative magnitudes and, since the exposures being compared were
Table 3. Apparent magnitudes of the LMXB counterparts.

\begin{tabular}{lcc}
\hline \hline Source & & \\
Filter & Exp (s) & Magnitude \\
\hline XTE J1637-498 & & \\
$U$ & 180 & $21.51 \pm 0.14$ \\
$B$ & 180 & $20.77 \pm 0.05$ \\
$V$ & 180 & $18.90 \pm 0.05$ \\
$R$ & 180 & $17.66 \pm 0.05$ \\
$I$ & 180 & $16.24 \pm 0.16$ \\
$J$ & 90 & $14.80 \pm 0.09$ \\
$H$ & 90 & $14.13 \pm 0.10$ \\
$K_{\mathrm{S}}$ & 90 & $13.55 \pm 0.07$ \\
$3.6 \mu \mathrm{m}$ & $\ldots$ & $12.87 \pm 0.09$ \\
$4.5 \mu \mathrm{m}$ & $\ldots$ & $12.51 \pm 0.10$ \\
$5.8 \mu \mathrm{m}$ & $\ldots$ & $12.41 \pm 0.17$ \\
$8.0 \mu \mathrm{m}$ & $\ldots$ & $12.28 \pm 0.24$ \\
\hline IGR J17379-3747 & & \\
$R$ & 180 & $21.5 \pm 0.3$ \\
$I$ & 180 & $20.0 \pm 0.4$ \\
$J$ & 90 & $18.3 \pm 0.1$ \\
$H$ & 90 & $\gtrsim 18.0$ \\
$K_{\mathrm{S}}$ & 90 & $\gtrsim 18.0$ \\
\hline IGR J17585-3057 & & \\
$K_{\mathrm{S}}$ & 54 & $14.18 \pm 0.05$ \\
$3.6 \mu \mathrm{m}$ & $\ldots$ & $11.38 \pm 0.05$ \\
$4.5 \mu \mathrm{m}$ & $\ldots$ & $10.71 \pm 0.05$ \\
$5.8 \mu \mathrm{m}$ & $\ldots$ & $10.07 \pm 0.05$ \\
$8.0 \mu \mathrm{m}$ & $\ldots$ & $9.30 \pm 0.03$ \\
\hline GX $9+1$ & 90 & $15.35 \pm 0.04$ \\
$K_{\mathrm{S}}$ & & \\
\hline & &
\end{tabular}

Notes. ${ }^{(\dagger)}$ For observations with 2 epochs, magnitudes were consistent so we present the weighted average and, since the exposures being compared were equal, the exposure of a single image.

equal, the quoted exposures are of a single image only. The equation, $i-I=(0.247 \pm 0.003)(R-I)$ (Jordi et al. 2006), was used to transform the cataloged $I$ magnitudes of the standard stars into $i$ magnitudes with which to calibrate the images. It was again used to transform the observed $i$ band magnitude of the object into appropriate $I$ magnitudes. Upper limits are approximated from the dimmest observable object in the region of interest.

To supplement our own observations, we utilise data from the Spitzer Space Telescope's (Werner et al. 2004) Galactic Legacy Infrared Mid-Plane Survey Extraordinaire (GLIMPSE; Benjamin et al. 2003), when available. GLIMPSE was carried out by the IRAC instrument (Fazio et al. 2004) aboard Spitzer, spans $65^{\circ}$ either side of the Galactic center up to $\pm 2-4^{\circ}$ in latitude and includes mosaic images and catalog entries at 3.6, 4.5, 5.8 and $8.0 \mu \mathrm{m}$.

The observed infrared magnitudes (Table 3) were first converted to flux densities, $F_{v}$, at frequency $v$, then to flux per filter, 


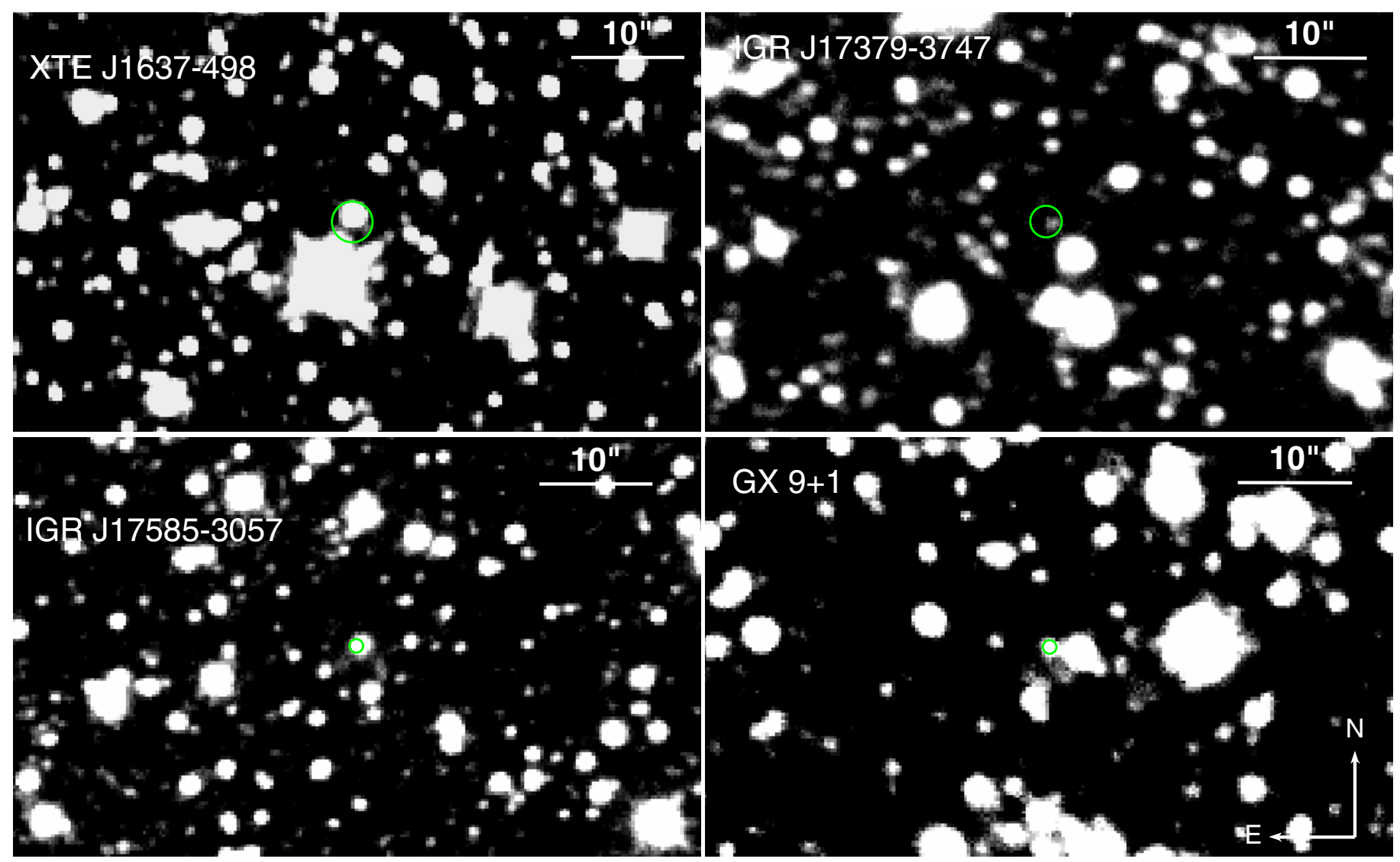

Fig. 1. NTT images $\left(60^{\prime \prime} \times 35^{\prime \prime}\right)$ of the fields of XTE J1637-498, IGR J17379-3747, IGR J17585-3057 and GX 9+1 with the X-ray 90\% position marked. IGR J17379-3747 is an EFOSC2 $R$ band image, while all others are SofI $K_{\mathrm{S}}$ band images; all have a common scaling and orientation.

$F_{\text {filter }}$ in units of photons $\mathrm{cm}^{-2} \mathrm{~s}^{-1}$. This is done via $F_{\text {filter }}=$ $1509.18896 F_{y}(\Delta \lambda / \lambda)$ where $\lambda$ and $\Delta \lambda$ are the effective wavelength and full width at half maximum of the filter in question. XSPEC compatible files, for SED fitting, are then produced from the flux per filter value using the FTOOL, flx2xsp.

\section{Results}

\subsection{XTE J1637-498}

The transient X-ray source, XTEJ1637-498, was first detected by the Rossi X-ray Timing Explorer (RXTE) at a $2-10 \mathrm{keV}$ flux of 2-4 mCrab; an outburst which lasted approximately from August 25 to September 52008 (Markwardt et al. 2008b). Follow up observations by the Swift X-ray Telescope (XRT) allowed the position to be refined (Table 1; Starling et al. 2008). Based on a power law fit (photon index $1.5 \pm 0.4$ ) of the XRT spectrum, Wijnands et al. (2008) suggested that the source was an LMXB, though they cautioned that another system type (i.e., high mass X-ray binary) could not be excluded. Starling et al. also noted that an optical source, consistent with 2MASS object J16370267-4951401, was detected in the $v$ band by the Swift UltraViolet and Optical Telescope (UVOT), within the XRT positional error. No further analysis of this source was published so its true classification remains unknown.

Within the X-ray positional error, we find that an optical and nIR source is detected in all filters (Fig. 1; best seeing was $\sim 0.95^{\prime \prime}$ in nIR filters and 1.0-1.1" in optical filters). From examination of our second epoch nIR images we derive a position of RA, Dec $=16: 37: 02.66-49: 51: 40.0\left( \pm 0.16^{\prime \prime}\right)$, consistent with 2MASS object J16370267-4951401, noted by Starling et al. (2008). We find no variability $(\lesssim 0.05 \mathrm{mag})$ of the counterpart

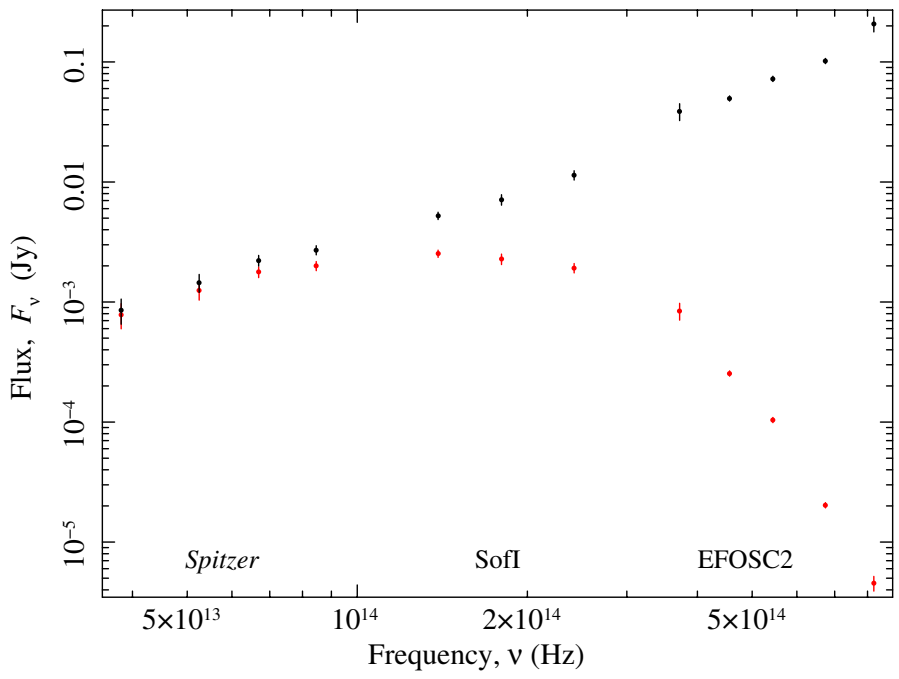

Fig. 2. Flux versus frequency plot for XTEJ1637-498: uncorrected (lower) and corrected (upper) for Galactic extinction in that direction of $E_{B-V}=2.143$.

between the two epochs so present only the average values (Table 3). We note that the observed magnitudes in $J, H$ and $K_{\mathrm{s}}$ are consistent with the cataloged 2MASS values; this is not surprising, given that the observations were obtained after the X-ray outburst had faded. Furthermore we note that this source is also tabulated in the GLIMPSE catalog (G335.4256-01.7828).

Figure 2 shows the spectral shape of the counterpart of XTE J1637-498, including the GLIMPSE data. Also plotted are 
the fluxes corrected for a Galactic extinction in that direction (Schlegel et al. 1998) of $E_{B-V}=2.143^{1}\left(A_{K} \sim 0.8, A_{V} \sim 7.1\right.$; Cardelli et al. 1989), though this should be considered as an upper limit. Though we are unable to find a single model - for any value of Galactic extinction - that fits both the NTT and GLIMPSE data, we find that the NTT data alone can be fit by black body radiation at a temperature of 3100-12800 K and corresponding extinctions of $0.4<E_{B-V}<2.0$. However, when extended to the Spitzer data, this model underestimates the flux at those wavelengths. In fact, the Spitzer data itself implies a power law $\left(F_{v} \propto v^{\alpha}\right)$ of spectral index $1.0<\alpha<1.7$ for the same range of extinctions as implied by the black body fit to the NTT data. A power law of similar slope is not a good fit to the NTT data alone or the NTT and Spitzer data combined $\left(\chi_{v}^{2} \gg 1\right)$. This implies that there is excess emission at the Spitzer-IRAC wavelengths (see Sect. 4), either due to intrinsic IR excess or an excess at the time of the Spitzer observations which, as catalog magnitudes, were not simultaneous with the NTT observations.

\subsection{IGR J17379-3747}

Outbursts from IGRJ17379-3747 ${ }^{2}$ were first reported from the INTErnational Gamma-Ray Astrophysics Satellite (INTEGRAL) by Chelovekov et al. (2006) with a fuller investigation of INTEGRAL and other observations presented in Chelovekov \& Grebenev (2010). Chelovekov et al. (2006) classified the source as an X-ray burster - a weakly magnetized, accreting neutron star in a LMXB system - on the basis of a type-I burst or a thermonuclear explosion, due to the unstable burning of $\mathrm{H}$ and $\mathrm{He}$ on the neutron star surface (Hoffman et al. 1978). During the 2008 outburst the X-ray position was refined to arcsecond precision (Table 1; Krimm et al. 2008) by the Swift-XRT.

At the X-ray position a new source is detected in our $i$ and $R$ images on both nights (Fig. 1; best seeing was $\sim 1.4^{\prime \prime}$ in nIR filters and $\sim 1.2^{\prime \prime}$ in optical filters). The source exhibits no variability $(<0.05,<0.1 \mathrm{mag}$ respectively) between the 2 epochs which are separated by 3 days (we present the average magnitudes). The lack of variability is not surprising since observations were taken after the 2008 September 2-21 X-ray outburst had faded (Markwardt et al. 2008a). The source is also detected in the second epoch $J$ band image but not in the first, though the magnitude limit on that night is consistent with the detected magnitude. We note that the counterpart is at the edge of the psf of a nearby, bright 2MASS object (J17375858-3746228; $\left.J=13.210, H=12.067, K_{\mathrm{s}}=11.743\right)$. There was no detections in the $U, B$ and $V$ bands and the lack of suitable optical standard stars on either night have lead us to omit their magnitude limits. The position of the optical/nIR counterpart was derived as RA, Dec $=17: 37: 58.77-37: 46: 19.9\left( \pm 0.32^{\prime \prime}\right)$ from the $R$ and $J$ band images. The spectral shape of the proposed OIR counterpart of IGR J17379-3747 (Fig. 3) is not particularly constraining but is consistent with stellar-like black body emission for a realistic range of temperatures, at extinctions up to the value for Galactic extinction in that direction (Schlegel et al. 1998) of $E_{B-V}=1.506^{1}\left(A_{K} \sim 0.5\right.$; Cardelli et al. 1989). This region is not covered by the GLIMPSE survey so we are unable to extend the SED redwards.

\footnotetext{
${ }^{1}$ Note that these extinctions should be treated with caution as estimates so close to the Galactic plane $(<5 \mathrm{deg})$ are unreliable.

2 While Chelovekov et al. (2006) and Chelovekov \& Grebenev (2010) refer to the source by differing IGR designations (IGR J17364-2711 and IGRJ17380-3749), herein we shall adhere to that of the INTEGRAL catalog (Bird et al. 2007, 2010).
}

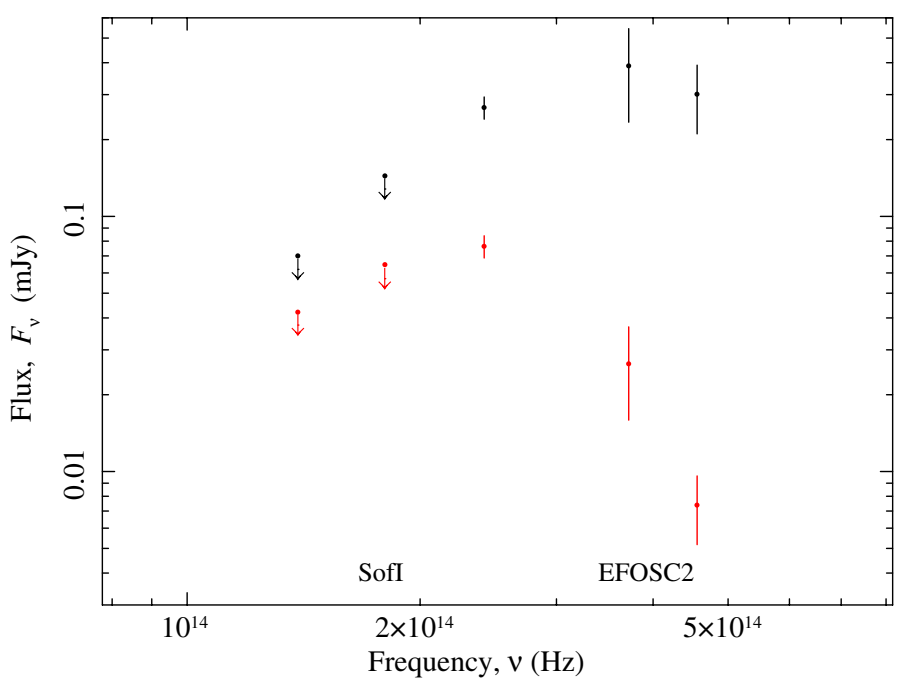

Fig. 3. Flux versus frequency plot for IGR J17379-3747: uncorrected (lower) and corrected (upper) for Galactic extinction in that direction of $E_{B-V}=1.506$.

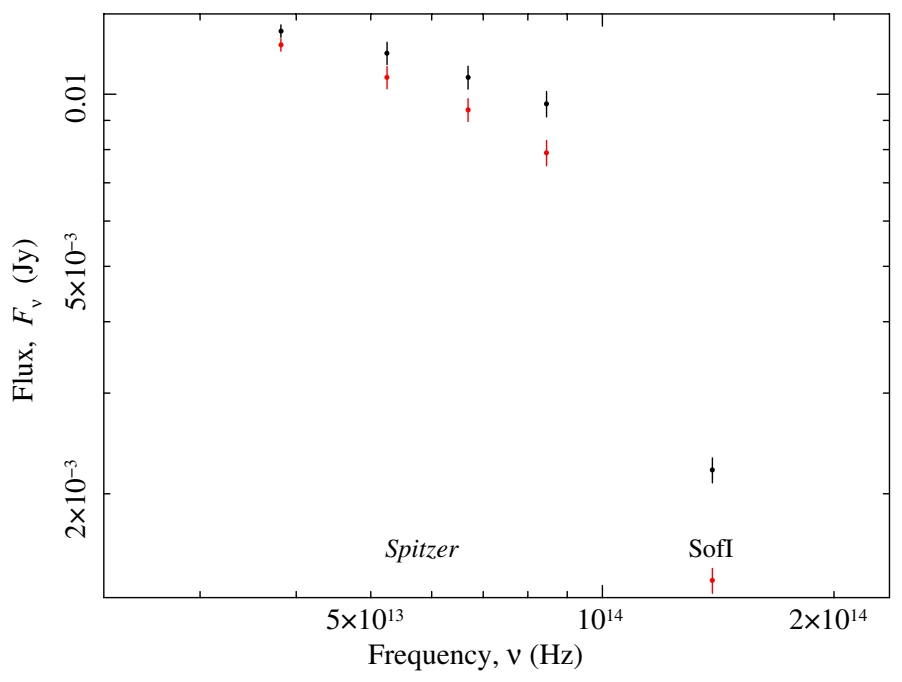

Fig. 4. Flux versus frequency plot for IGR J17585-3057: uncorrected (lower) and corrected (upper) for Galactic extinction in that direction of $E_{B-V}=1.302$.

\subsection{IGR J17585-3057}

Detected by INTEGRAL (Bird et al. 2007, 2010), IGR J17585-3057 is assumed to be a LMXB based on Galactic position and the lack of an alternative classification (Revnivtsev et al. 2008). Monitored as part of both the INTEGRAL all-sky survey in hard X-rays (Krivonos et al. 2007) and the Swift-BAT 58-Month Hard X-ray Survey (Baumgartner et al. 2010) there has been no evidence of flux variation. No further analysis of this source was published so its true classification remains unknown, though an accurate X-ray position was obtained by Chandra (Table 1; M. Revnivtsev, 2011 priv. comm.).

At the X-ray position we find a bright, $K_{\mathrm{S}}=14.183 \pm 0.05$ counterpart in our single SofI image of the field (Fig. 1; seeing $\left.\sim 0.8^{\prime \prime}\right)$ and we derive a position of RA, Dec $=17: 58: 29.83$ $-30: 57: 01.6\left( \pm 0.1^{\prime \prime}\right)$. This coincides with a GLIMPSE catalog source (G359.6862-03.4261), the magnitudes of which we reproduce in Table 3. From the SED (Fig. 4), it is clear that the extrapolation of the Spitzer data overestimates the $K_{\mathrm{S}}$ magnitude significantly. In fact the Spitzer data implies a spectral 
index of $\alpha=-0.37 \pm 0.10$ (when optical extinction is set to that of Galactic extinction in that direction, Schlegel et al. 1998, $E_{B-V}=1.302^{1} ; A_{K} \sim 0.4$; Cardelli et al. 1989) but then drops by almost an order of magnitude, requiring a steepening to $\alpha \gtrsim 5$ to agree with the $K_{\mathrm{S}}$ flux. This implies either intrinsic excess emission at the Spitzer-IRAC wavelengths or it is possible that the source is variable, though without multiple photometric observations, we cannot test this.

\section{4. $G \times 9+1$}

The persistent X-ray source GX9+1, a.k.a. SgrX-3, was initially discovered by sounding rockets (Bradt et al. 1968; Mayer et al. 1970) and later confirmed by the first dedicated X-ray astronomy satellite, Uhuru (as 2U 1758-20; Giacconi et al. 1971, 1972). Thereafter, it was observed by most other X-ray missions which revealed that the source is likely an "Atoll" source, a class of neutron star LMXB (e.g., Langmeier et al. 1985; Hasinger \& van der Klis 1989; Schulz et al. 1989). Previous searches for an optical or nIR counterpart (Gottwald et al. 1991; Naylor et al. 1991) did not yield any plausible candidates due to the lack of an accurate X-ray position, which is now available from Chandra observations (Table 1; M. Revnivtsev, 2011, priv. comm.).

At the X-ray position we detect a $K_{\mathrm{S}}=15.35 \pm 0.04$ source in our single SofI image of the field (Fig. 1; seeing $\sim 1.4^{\prime \prime}$ ) and we derive a position of RA, Dec $=18: 01: 32.15-20: 31: 46.2$ $\left( \pm 0.16^{\prime \prime}\right)$. We note that there is also a source directly to the West which appears in the GLIMPSE (G009.0755+01.1545) and 2MASS $\left(\mathrm{J} 18013196-2031464 ; K_{\mathrm{S}}=13.263 \pm 0.055\right)$ catalogs but we find that it is $>6 \sigma$ from the X-ray position and is hence likely unrelated to GX 9+1.

\section{Discussion and conclusions}

To approximate the probability of a chance superposition of the $90 \%$ X-ray positions with random sources in the respective fields, we calculate $P \approx \rho_{N} \times A_{\text {Err }}$; where $\rho_{N}$ is the surface area number density of observed sources down to the limiting magnitude and $A_{\mathrm{Err}}$ is the area of the X-ray positional error. The probability of chance coincidence for XTE J1637-498 and IGR J17379-3747 are quite high at $86 \%$ and $28 \%$ respectively, due to a large number of sources in the deep fields but more significantly, the radius of the X-ray positional errors. The importance of sub-arcsecond positions from e.g. Chandra is further illustrated by the significantly lower probabilities of IGR J17585-3057 and GX 9+1 (8\% and 7\%) which have comparable number densities to the previous two fields but much more accurate X-ray positions. The probability of chance superposition could be reduced further if we limited our search to sources of specific magnitude ranges but due to a lack of information on these sources, we make no such assumption.

On the basis of positional coincidence with the arcsecond accurate X-ray positions, we suggest unambiguous (in so far as there is only one per source) counterpart candidates for four LMXBs near the Galactic centre. We caution that given the probability of chance superpositions in these crowded fields, the associations are tentative and require further investigations of e.g., spectral type, variability, distance, to confirm the associations with the X-ray sources. The spectral shapes of the counterpart candidates are not particularly constraining but, in general, could be consistent with stellar-like black body emission in the optical/nIR but with an excess at the longer, Spitzer, wavelengths, as for XTEJ1637-498. The excess could be due to warm circumbinary dust, likely related to the formation of the compact object (e.g., Rahoui et al. 2010) or to non-thermal, synchrotron jet emission (e.g., Gelino et al. 2010). Further, possibly simultaneous, photometric and spectroscopic observations are required to disentangle the different emission components and identify the spectral type of the companion star, if it is detected over the other possible emission mechanisms.

Acknowledgements. We thank the referee for the constructive comments and M. Revnivtsev for supplying Chandra positions for two of the sources in our study. This work was supported by the Centre National d'Études Spatiales (CNES) and is based on observations obtained with MINE: the Multi-wavelength INTEGRAL NEtwork. This research has made use of NASA's Astrophysics Data System and the VizieR catalogue access tool, CDS, Strasbourg. IRAF is distributed by the National Optical Astronomy Observatory, which is operated by the Association of Universities for Research in Astronomy (AURA) under cooperative agreement with the National Science Foundation. GAIA was created by the now closed Starlink UK project funded by the Particle Physics and Astronomy Research Council (PPARC), and has been more recently supported by the Joint Astronomy Centre Hawaii funded by PPARC and now its successor organisation, the Science and Technology Facilities Council (STFC).

\section{References}

Baumgartner, W. H., Tueller, J., Markwardt, C., \& Skinner, G. 2010, in AAS/High Energy Astrophysics Division \#11, BAAS, 42, 675

Benjamin, R. A., Churchwell, E., Babler, B. L., et al. 2003, PASP, 115, 953

Bird, A. J., Malizia, A., Bazzano, A., et al. 2007, ApJS, 170, 175

Bird, A. J., Bazzano, A., Bassani, L., et al. 2010, ApJS, 186, 1

Bradt, H., Naranan, S., Rappaport, S., \& Spada, G. 1968, ApJ, 152, 1005

Cardelli, J. A., Clayton, G. C., \& Mathis, J. S. 1989, ApJ, 345, 245

Charles, P. A., \& Coe, M. J. 2006, in Compact Stellar X-ray Sources, ed. W. Lewin \& M. van der Klis, Cambridge Univ. Ser., 39, 215

Chelovekov, I. V., \& Grebenev, S. A. 2010, Astron. Lett., 36, 895

Chelovekov, I. V., Grebenev, S. A., \& Sunyaev, R. A. 2006, Astron. Lett., 32, 456

Fazio, G. G., Hora, J. L., Allen, L. E., et al. 2004, ApJS, 154, 10

Gelino, D. M., Gelino, C. R., \& Harrison, T. E. 2010, ApJ, 718, 1

Giacconi, R., Kellogg, E., Gorenstein, P., Gursky, H., \& Tananbaum, H. 1971, ApJ, 165, L27

Giacconi, R., Murray, S., Gursky, H., et al. 1972, ApJ, 178, 281

Gottwald, M., Steinle, H., Pietsch, W., \& Graser, U. 1991, A\&AS, 89, 367

Grimm, H.-J., Gilfanov, M., \& Sunyaev, R. 2002, A\&A, 391, 923

Hasinger, G., \& van der Klis, M. 1989, A\&A, 225, 79

Hoffman, J. A., Marshall, H. L., \& Lewin, W. H. G. 1978, Nature, 271, 630

Jordi, K., Grebel, E. K., \& Ammon, K. 2006, A\&A, 460, 339

Krimm, H. A., Kennea, J. A., Evans, P. A., \& Markwardt, C. B. 2008, The Astronomer's Telegram, 1714

Krivonos, R., Revnivtsev, M., Lutovinov, A., et al. 2007, A\&A, 475, 775

Landolt, A. U. 1992, AJ, 104, 340

Langmeier, A., Sztajno, M., Truemper, J., \& Hasinger, G. 1985, Space Sci. Rev., 40, 367

Markwardt, C. B., Krimm, H., Wijnands, R., \& Swank, J. H. 2008a, The Astronomer's Telegram, 1709

Markwardt, C. B., Pereira, D., \& Swank, J. H. 2008b, The Astronomer's Telegram, 1699

Mayer, W., Bradt, H. V., \& Rappaport, S. 1970, ApJ, 159, L115

Monet, D. G., Levine, S. E., Canzian, B., et al. 2003, AJ, 125, 984

Naylor, T., Charles, P. A., \& Longmore, A. J. 1991, MNRAS, 252, 203

Persson, S. E., Murphy, D. C., Krzeminski, W., Roth, M., \& Rieke, M. J. 1998, AJ, 116, 2475

Rahoui, F., Chaty, S., Rodriguez, J., et al. 2010, ApJ, 715, 1191

Revnivtsev, M., Lutovinov, A., Churazov, E., et al. 2008, A\&A, 491, 209

Russell, D. M., \& Fender, R. P. 2010, in Black Holes and Galaxy Formation (Nova Science Publ.), in press [arXiv: 1001.1244]

Russell, D. M., Fender, R. P., Hynes, R. I., et al. 2006, MNRAS, 371, 1334

Schlegel, D. J., Finkbeiner, D. P., \& Davis, M. 1998, ApJ, 500, 525

Schulz, N. S., Hasinger, G., \& Truemper, J. 1989, A\&A, 225, 48

Skrutskie, M. F., Cutri, R. M., Stiening, R., et al. 2006, AJ, 131, 1163

Starling, R., Evans, P., \& Wijnands, R. 2008, The Astronomer's Telegram, 1704

Stetson, P. B. 1987, PASP, 99, 191

van Paradijs, J., \& McClintock, J. E. 1994, A\&A, 290, 133

Werner, M. W., Roellig, T. L., Low, F. J., et al. 2004, ApJS, 154, 1

Wijnands, R., Linares, M., Degenaar, N., \& Markwardt, C. B. 2008, The Astronomer's Telegram, 1700

Zolotukhin, I. Y., \& Revnivtsev, M. G. 2011, MNRAS, 411, 620 